\title{
Experimental investigation on convective Heat Transfer Enhancement by EHD
}

Merouane Hamdi, Michel Havet, Olivier Rouaud GEPEA (CNRS UMR 6144), ONIRIS, rue de la Geraudière, BP 82225, 44322 Nantes, France

Dominique Tarlet Laboratoire de Thermocinétique de Nantes (CNRS UMR 6607),

Polytech Nantes, rue C. Pauc, BP 50609, 44306 Nantes, France

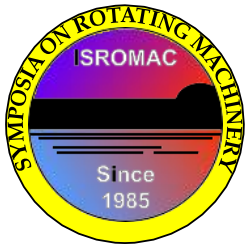

Long Abstract

\section{Introduction}

One of the most promising techniques to enhance convective heat transfer in air is based on the use of electric fields to induce secondary flows (or ionic wind). The ions which are propelled by the Columbic force transfers momentum to neutral fluid molecules which results in the bulk movement of air, causing an interaction between the ionic wind and the primary air flow (EHD combined flow). The EHD combined flow increases the convective heat transfer coefficient on the plate surface [3], [1]. Many questions regarding the optimization of the operating parameters still remain unanswered as reported by [1],[2]. In this context, we are interested in the forced convection heat transfer enhancement by the ionic wind produced by corona discharge (wire to plate) in a channel.

\section{Methods}

An experimental rig is designed specifically for analyzing firstly the convective exchange at the bottom wall in a channel [Fig 1]. A series of experiments were performed in a horizontal air flow channel equipped with different arrangements of wire-electrodes suspended above a conductive plate. These electrodes were arranged either parallel or perpendicular to the direction of primary air flow. Measurements allow determination of the optimal configurations for electrodes location and operating system (voltage $V$, air velocity $U_{0} \ldots$ ) taking into account the energy consumption and the heat transfer distribution [Fig 1.a].

Secondly, experiments based on Particle Image Velocimetry (PIV) were conducted on selected configurations. [Fig 1.b]

- Several electric power $=$ Voltage $(k V) \times$ Current $(\mu A)$

- 3 wire-to-plate distances $(\mathrm{H}=2,4,6 \mathrm{~cm})$

- 3 inlet channel velocities $\left(U_{0}=0,0.3,0.6,1 \mathrm{~m} / \mathrm{s}\right)$
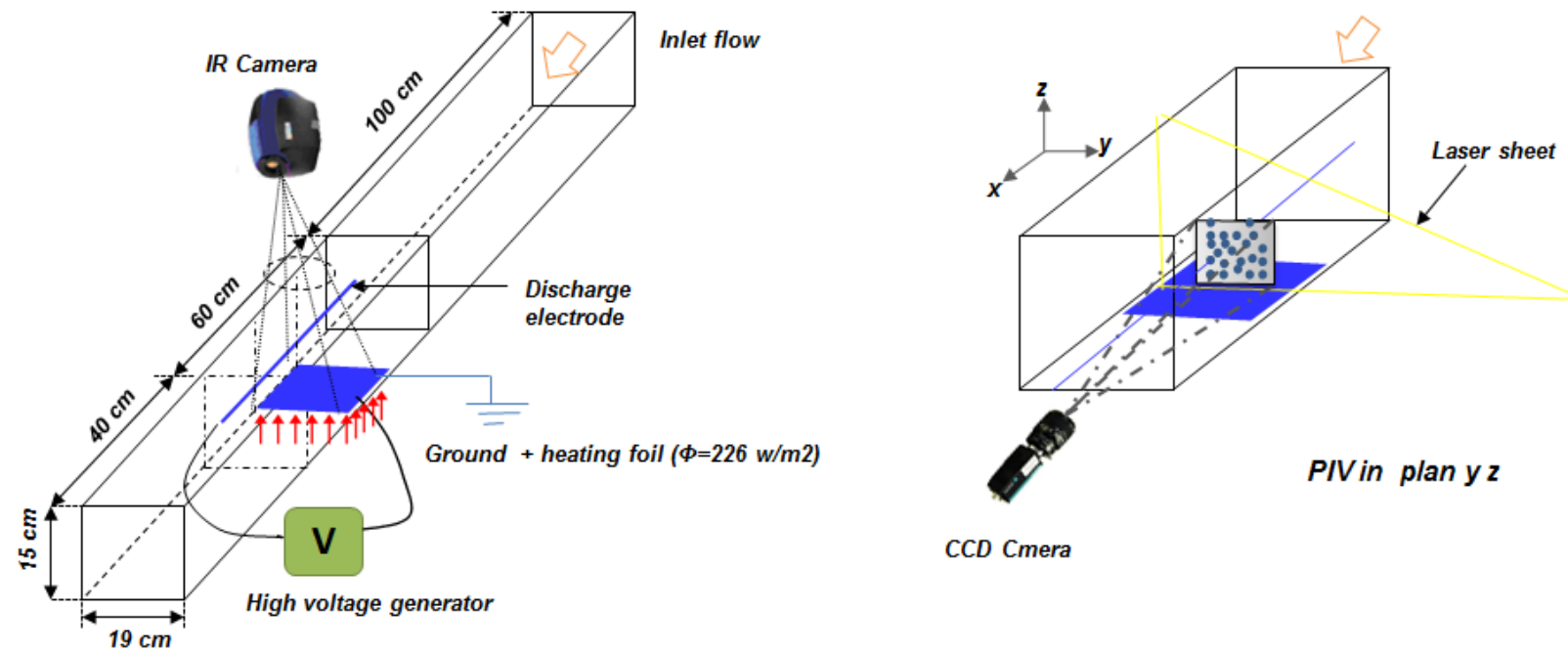

CCD Cmera

Figure 1. Schematic of the experimental set-up and wire-electrode placement 


\section{Results}

- In most cases results show that heat transfer enhancement due to EHD is significant at low Reynolds numbers $\left(\mathrm{U}_{0}=0.3 \mathrm{~m} / \mathrm{s}\right)$.

-Low H: high local heat transfer due to the plane-jet like air flow but low mean heat transfer

-High $\mathrm{H}$ : better expansion of the plane jet, but the low velocities resulted in lower mean heat transfer

-The most significant increase in heat transfer resulted in a single wire-electrode arranged perpendicular to the air flow direction (8x higher compared to forced convection without EHD):

-Optimal mean heat transfer coefficient was obtained for $\mathrm{H}=4 \mathrm{~cm}$

-Counter rotating vortex pair generated by the combination of ionic wind and primary channel air flow.

-For cases of more than one parallel arranged wire-electrode the heat transfer enhancement was slightly lower (6x) but more wide-spread over the conductive plate.

-Interactions between the vortices generated by the combination of the secondary flow and the primary air flow are well understood. The impact of these structures and velocity gradients on the local and average heat transfer is evaluated [Fig 2].

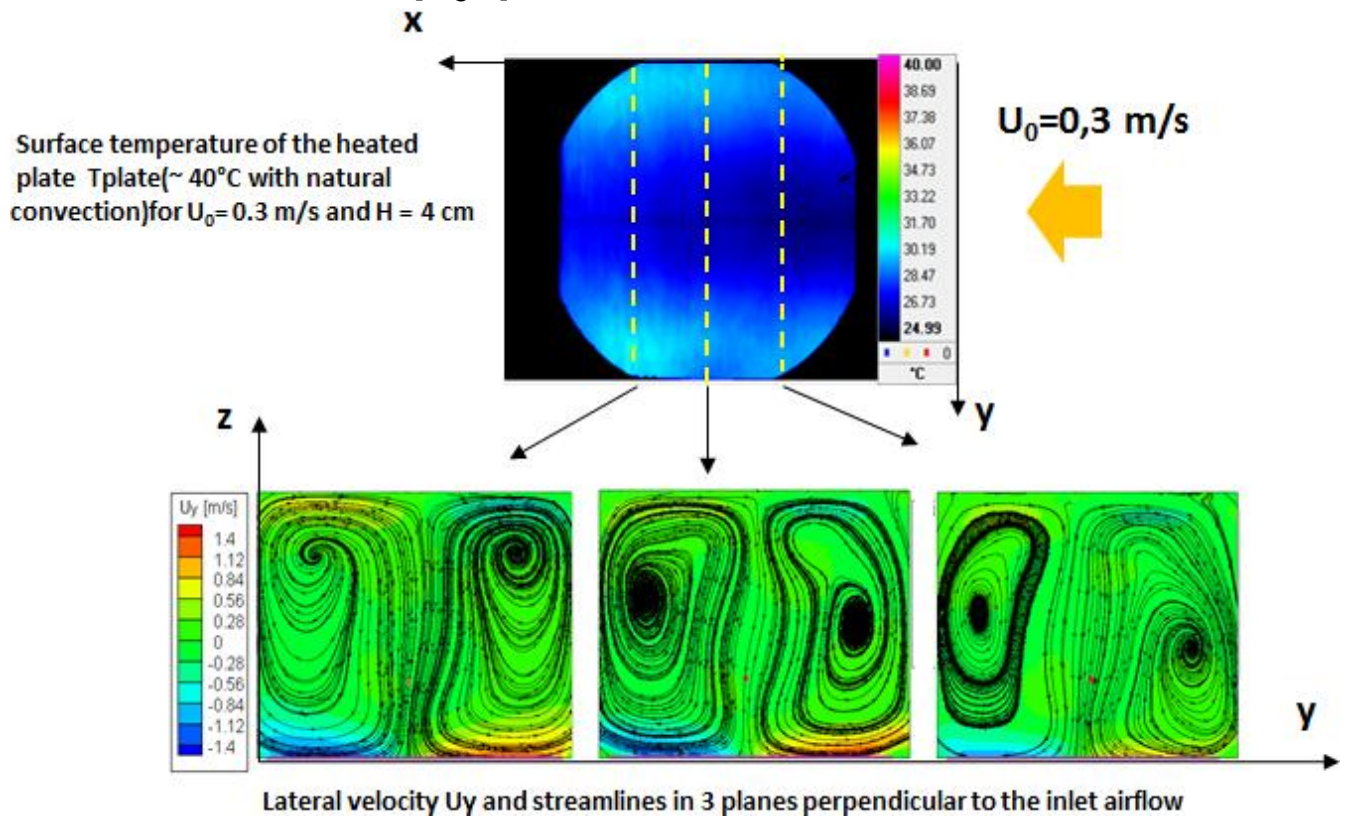

Figure 2. Schematic of the surface temperature from IR camera and PIV visualization structures and velocity gradients

\section{Acknowledgements}

The authors gratefully acknowledge the support of the Pays de Loire program for outstanding research in the field of energetics (PERLE2).

\section{References}

[1] F.C. Lai. Effects of buoyancy on electro-hydrodynamic enhanced forced convection in a horizontal channel, J. Thermophys. Heat Transfer 12 (3) 431-436,1998.

[2] Tada, Y. A. Takimoto, Y, Hayashi. Heat transfer enhancement in a convective field by applying ionic wind", J. Enhanced Heat Transfer, Vol. 4, pp. 71-86,1997.

[3] Ould Ahmedou, S.A Havet, M. Effect of process parameters on the EHD airflow Journal of
Electrostatics,
Vol.
67,
pp.
222-227,
2009 
